\title{
On Computing ELECTRE's Credibility Indices under Partial Information
}

\author{
LUIS C. DIAS ${ }^{a, b, *}$ and JOÃO N. CLíMACO ${ }^{a, b}$ \\ a Faculty of Economics, University of Coimbra, Av. Dias da Silva, Coimbra, Portugal \\ b INESC, R. Antero de Quental 199, 3000-033 Coimbra, Portugal
}

\begin{abstract}
The ELECTRE family of decision aid methods is a well-known approach to help decision makers (DMs) advance in a decision process. Among the most recent methods in the family, ELECTRE IS, III, and TRI compute fuzzy indices for the credibility of a given action outranking some other. We consider the case when the DMs are unsure which values each parameter should take, which may result from insufficient, imprecise or contradictory information, as well as from different preferences among a group of DMs. In the framework of a robustness analysis approach, where DMs provide only partial information on the parameter values (through constraints on acceptable combinations), we study how to find whether an outranking among two actions in an ELECTRE method is robust. In this context, we study the resulting nonlinear problems of optimizing a credibility index (under the type of constraints that we consider to appear in practice) and present some examples. Copyright (C) 1999 John Wiley \& Sons, Ltd.
\end{abstract}

KEY WORDS: ELECTRE methods; partial information; robustness analysis

\section{INTRODUCTION}

As B. Roy (1997) has noted, the goal of decision aid has shifted from finding the right solution to a problem, to trying to provide some support to the decision makers (DMs) that will allow them to advance in a decision process. To accomplish this goal, answers are drawn from algorithms working on some mathematical model. These models, and sometimes the behaviour of the algorithms, are characterized by several parameters, whose nature is often subjective (we consider parameters in a broad sense, including input usually referred to as 'data', input concerning the values and beliefs of the DMs, input for tuning an algorithm, etc.). However, it is unrealistic to expect that the DMs are able to determine the value that each parameter should take. This is particularly noticed when the decision aid concerns the multicriteria evaluation of actions, to the extent that (see also on this subject Roy and Bouyssou, 1989; French, 1995):

- the performance of the actions at each evaluation criterion may depend on the future state

\footnotetext{
* Correspondence to: Universidade de Coimbra, Faculdade de Economia, Av. Dias da Silva, 165, 3000-033 Coimbra, Portugal. Tel.: + 35139 790500; e-mail: idias@inescc.pt
}

of some variables, it may result from aggregating several aspects with an impact on the criterion (i.e. there may exist some arbitrariness in constructing parts of the model), and it may result from measuring instruments or statistics (which usually involve some imprecision);

- many parameters, e.g. those defining the importance of the criteria, have no objective existence (they are inherent to the decision aid method); moreover, they reflect the subjective values of the DMs, which they may find difficult to express and that may change over time; - in either case, there may be several DMs in a group decision setting who may not entirely agree on the values that each parameter should take.

For these reasons, any combination of values for the parameters should be seen as a 'working hypothesis' that allows the decision process to advance. The problem facing the DMs is that different 'working hypotheses' may lead to different results. There are two approaches that acknowledge this difficulty and complement each other: sensitivity analysis and robustness analysis (as presented by Roy and Bouyssou, 1993).

Sensitivity analysis determines how much may each parameter vary without leading to a different 
result. Although useful in many circumstances (e.g. Henggeler Antunes and Clímaco, 1992) it has the disadvantage of requiring an 'estimated' value for each parameter and focusing on the corresponding solution. It ignores other potentially interesting results that would have been found with different parameter values. Furthermore, the analysis is often performed on a single parameter at a time, thus ignoring possible interdependencies among them.

Robustness analysis considers all the results compatible with the admissible combinations of values for the parameters. Roy (1997) presented a framework defining the concept of robust conclusion as a formalized premise that is true for all these combinations. On a different framework, Vincke (1997) proposes a formalism to define the concepts of robust solutions and robust methods. His concept of robust solution does not require it to remain unchanged for all admissible combinations of parameter values, provided that none of these leads to a solution that contradicts the first one (in some formalized and problem-dependent manner). Finally, Kouvelis and Yu (1997) define robust solution (in the context of optimization problems) as the solution with the best worst-case behaviour.

Let $T$ represent the set of all acceptable combinations of parameter values. We will consider that the DMs are able to agree on which constraints $T$ should obey to. To ask for such constraints is, of course, less demanding (to the DMs) than requiring a precise value for each parameter. Along with other authors (see list of references), we will refer to this situation as one of partial information. The DMs may start with a set $T$ not too constrained and then progressively reduce $T$ as their convictions become stronger or consensus emerges.

We will use Roy's definition of a robust conclusion, although we introduce a further distinction that we deem useful when using decision aid methods. Let us define:

- An absolute robust conclusion is a premise intrinsic to one of the actions, which is valid for every combination in $T$. For instance, in an additive aggregation model one may check the robustness of the absolute conclusion 'the value of action $x$ is greater than 0.7 '.

- A (relative) binary robust conclusion is a premise concerning a pair of actions, which is valid for every combination in $T$. For instance,
' $x$ dominates $y$ ' or ' $x$ outranks $y$ with credibility greater than 0.7 ' are possible binary robust conclusions.

- A (relative) unary robust conclusion is a premise concerning one action but relative to others, which is valid for every combination in $T$. For instance, ' $x$ is non-dominated' or ' $x$ is among the three top actions in a ranking' are possible unary robust conclusions.

The ELECTRE family of methods (e.g. see Roy and Bouyssou, 1993; Maystre et al., 1994) is a well-known approach concerning the evaluation of a discrete set of potential according to multiple criteria. These methods are based on the outranking concept (Roy, 1990): first, they build an outranking relation on a set of actions; afterwards, they exploit this relation for decision aid. The methods ELECTREs IS, III and TRI (for details see Roy and Bouyssou, 1993; Maystre et al., 1994) are among the most recent in the family. When a pair of actions $\left(a_{1}, a_{2}\right)$ are compared, these methods indicate an index $\sigma\left(a_{1}, a_{2}\right)$ stating the credibility of affirming that ' $a_{1}$ outranks (is at least as good as) $a_{2}$ ', instead of providing a crisp 'outranks'/'does not outrank' output. This index varies in $[0,1]$ : the higher it is, the most credible is the outranking of $a_{1}$ over $a_{2}$. The set of these indices for the all the pairs of actions that are being compared constitutes a fuzzy outranking relation (Roy, 1990).

This work focuses on binary robust conclusions concerning credibility indices in ELECTRE methods. We show how to compute the range of variation for such an index, given partial information on the parameter values (a set $T$ ). This is important, to the extent that these indices are the basis of some of the most popular ELECTRE methods. In the section 'Illustrative Examples' we illustrate how to find robust conclusions concerning interesting pairs of actions (possibly after ranking the actions using some method) and also how to exploit the credibility index range in the context of accept/reject decisions. The latter approach can be extended to find robust conclusions concerning the exploitation procedure of the ELECTRE TRI sorting method (see Dias and Clímaco, 1998b). Besides the identification of robust conclusions, this approach is also useful to discover which conclusions are more affected by the fact that information is partial.

The next section presents earlier approaches, showing how optimization may be used to check 
the robustness of a conclusion. In the section 'Computing ELECTRE's Credibility Index' we briefly overview the computation of ELECTRE's credibility indices and in 'Constraints of the Parameter Values' we characterize the set $T$ of partial information. In 'Computing the the Variation Interval of a Credibility Index' we discuss how to maximize or minimize a credibility index. The section 'Illustrative Examples' presents some examples on finding robust conclusions. We conclude with a summary and streams for future research. In the Appendix, we prove some results concerning the credibility index function.

\section{COMMON APPROACHES TO COPE WITH PARTIAL INFORMATION}

The problem of coping with partial information has been studied for a long time. Fishburn (1964) has studied the problem of choice under risk when there is partial information on the probabilities of each event. An analogous situation is that of partial information on the importance (weight) of each criterion when using a multicriteria additive aggregation model (e.g. see Kirkwood and Sarin, 1985).

In the context of additive value functions, optimization may be used to cope with partial information on the importance of each criterion, when this information is provided as a set of linear constraints delimiting a polytope $T$. An action 'dominates' another (in partial information terminology) if it has higher or equal aggregated value for every $t \in T$ (and strictly higher for some $t \in T$ ). Such binary conclusions may be tested using linear programming. Linear programs may also be used to test unary conclusions referring to whether an action is 'non-dominated' or whether it is 'optimal' (in the sense that it has the greatest aggregated value). For a review of these concepts and typical approaches refer to Hazen (1986), Weber (1987) or Rios Insua and French (1991). These ideas may be used in any context where actions are evaluated according to a value provided by an aggregation function. For instance, Dias and Clímaco (1998a) use these concepts in the context of a shortest path problem.

Kampke (1996) presents an approach based on mixed-integer programming to find the range of variation of an action's position in a ranking. In our terminology, this may be done to test the robustness of unary conclusions. It is based on the UTA method for inferring a multi attribute value function from holistic judgements that are transformed into constraints.

On considering ELECTRE methods, we can mention Roy's suggestion of testing the robustness of a conclusion in a finite number of sample points in $T$ (see e.g. Roy and Bouyssou, 1993; Roy, 1997). These points would be those admissible in the Cartesian product of a finite number of sets (one per parameter), each containing a few values (e.g. maximum, central and minimum) for the respective parameter. This is simpler than optimization and may provide an idea of the robustness of a conclusion. However, it may be insufficient if parameters are interdependent. For instance, let the shaded areas in Figure 1 represent the admissible combinations of values (accounting for interdependencies) for two parameters. In this example, A, B,..., I are potential sampling points, but only $\mathrm{C}, \mathrm{E}, \mathrm{F}$ and $\mathrm{G}$ are feasible. These points define the inner shaded area, which is a poor representation of the initial set. Moreover, if there are non-linearities present when evaluating each point, results for non-extreme points such as $\mathrm{P}$ are not guaranteed to belong to the set of results induced by points $\mathrm{C}, \mathrm{E}, \mathrm{F}$ and $\mathrm{G}$.

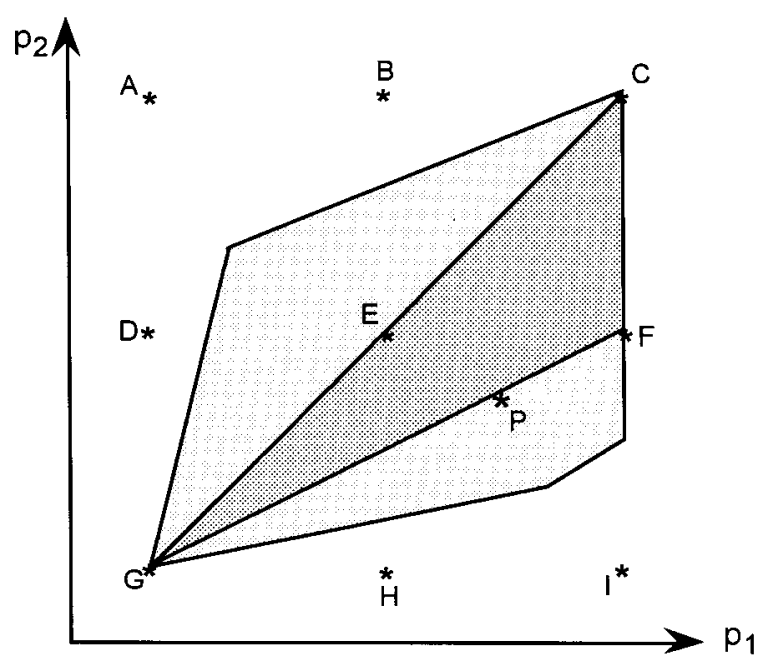

Figure 1. The shaded areas represent $T$, whereas points A, B, C, . . G, H, I represent potential sampling points. Only $\mathrm{C}, \mathrm{E}, \mathrm{F}$ and $\mathrm{G}$ are feasible, hence the sampling is a poor representation. 


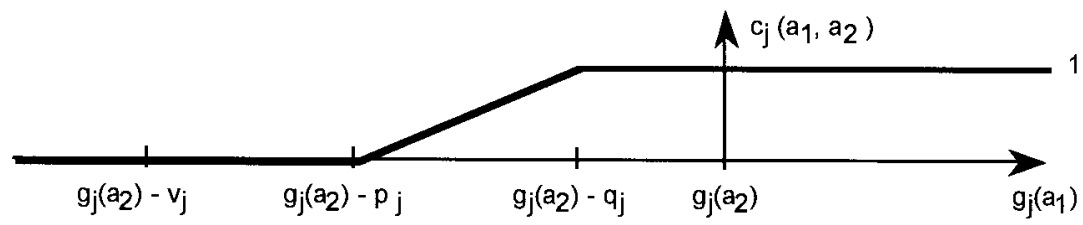

Figure 2. Single-criterion concordance index.

Optimization can be used to obtain more accurate information about the robustness of conclusions in ELECTRE methods, at the cost of demanding more computational power. This work focuses on conclusions that are the basis of many methods: to find, given a pair of actions $\left(a_{1}, a_{2}\right)$, whether ' $a_{1}$ outranks $a_{2}$ ' or ' $a_{1}$ does not outrank $a_{2}$ ' are robust. This amounts to determine the credibility index's range of variation under partial information.

\section{COMPUTING ELECTRE'S CREDIBILITY INDEX}

The first phase of ELECTRE is to compute credibility indices for ordered pairs $\left(a_{1}, a_{2}\right)$. Index $\sigma\left(a_{1}, a_{2}\right)$ measures the credibility of the statement ' $a_{1}$ outranks (is at least as good as) $a_{2}$ ', also represented as $a_{1} S a_{2}$. This section shows how to compute this index for a given combination of parameters (for more detail see Roy, 1990; Roy and Bouyssou, 1993).

Let there be $n$ pseudo-criteria. The $j$-th criterion $(j=1, \ldots, n)$ is characterized by an importance coefficient $k_{j}$, an indifference threshold $q_{j}$, a preference threshold $p_{j}$ and a veto threshold $v_{j}$. These thresholds may not be negative and $v_{j} \geq p_{j} \geq q_{j}$. For simplicity of notation we assume these thresholds do not depend on the actions' performances, but this work could be easily extended if this were not the case. Let $g_{j}\left(a_{1}\right)$ and $g_{j}\left(a_{2}\right)$ denote the performance on the $j$-th criterion of the actions $a_{1}$ and $a_{2}$, respectively.

Let $\Delta_{j}(j=1, \ldots, n)$ represent the advantage of $a_{1}$ over $a_{2}$ at the $j$-th criterion:
The concordance index concerning the $j$-th criterion and the hypothesis ' $a_{1} S a_{2}$ ' is (see Figure 2):

$$
c_{j}\left(a_{1}, a_{2}\right)= \begin{cases}1, & \text { if } \Delta_{j} \geq-q_{j} \\ 0, & \text { if } \Delta_{j}<-q_{j} . \\ \left(p_{j}+\Delta_{j}\right) /\left(p_{j}-p_{j}\right), & \text { otherwise }\end{cases}
$$

The global concordance index aggregates the $n$ single-criterion concordance indices as a weighted sum using the importance coefficients:

$c\left(a_{1}, a_{2}\right)=\sum_{j=1}^{n} k_{j} c_{j}\left(a_{1}, a_{2}\right)$,

$$
\text { where we assume that } \sum_{j=1}^{n} k_{j}=1 \text {. }
$$

The discordance index concerning the $j$-th criterion and the hypothesis ' $a_{1} S a_{2}$ ' is (see Figure 3):

$d_{j}\left(a_{1}, a_{2}\right)= \begin{cases}0, & \text { if }-\Delta_{j} \leq p_{j} \\ \left(-\Delta_{j}-p_{j}\right) /\left(v_{j}-p_{j}\right), & \text { if } p_{j}<-\Delta_{j} \leq v_{j} . \\ 1, & \text { if }-\Delta_{j}>v_{j}\end{cases}$

Finally, the discordance indices and the global concordance index are combined to yield the credibility index for the hypothesis ' $a_{1} S a_{2}$ ':

$$
\sigma\left(a_{1}, a_{2}\right)=c\left(a_{1}, a_{2}\right) \prod_{\substack{j \in\{1, \ldots, n\}: \\ d_{j}\left(a_{1}, a_{2}\right)>c\left(a_{1}, a_{2}\right)}} \frac{1-d_{j}\left(a_{1}, a_{2}\right)}{1-c\left(a_{1}, a_{2}\right)} .
$$

Notice that whenever there is a criterion (let $j$ be its index) where $\Delta_{j} \leq-v_{j}$, then $d_{j}\left(a_{1}, a_{2}\right)$ equals 1 and $\sigma\left(a_{1}, a_{2}\right)$ becomes null. During an exploitation phase, this binary valued relation may be

$\Delta_{j}=\left\{\begin{array}{ll}g_{j}\left(a_{1}\right)-g_{j}\left(a_{2}\right), & \text { if the } j \text {-th criterion is to be maximized (the more the better) } \\ g_{j}\left(a_{2}\right)-g_{j}\left(a_{1}\right), & \text { if the } j \text {-th criterion is to be minimized }\end{array}\right.$. 
transformed into a crisp relation by introducing a cut threshold $\lambda$ :

$a_{1} S a_{2}$ if $\sigma\left(a_{1}, a_{2}\right) \geq \lambda$.

\section{CONSTRAINTS ON THE PARAMETER VALUES}

Let us consider that there is partial information on the values of the parameters $k_{j}, q_{j}, p_{j}$ and $v_{j}$ $(j=1, \ldots, n)$, on the cut threshold $\lambda$ and on the performance of the actions. We assume that partial information is given as a set of bounds and linear constraints on the parameter values. These bounds and constraints may be directly provided by the DMs or inferred through a questioning protocol, as in Mousseau (1993).

We now provide a characterization of the resulting polytope $T$, accounting for the reasonable type of constraints that may be useful in practice (some types of parameters may be varied independently of others). For instance, a constraint such as $k_{1} \geq k_{2}$ is reasonable, whereas the constraint $k_{1}+\lambda \geq q_{1}$ is not. First of all, the variation of the cut threshold $\lambda$ should not depend on any other parameter, hence we assume it will only appear on a constraint:

$\lambda \in\left[\lambda_{\text {min }}, \lambda_{\text {max }}\right]$.

Indifference and preference thresholds are local to each criterion and inter-criteria comparisons of these thresholds have no meaning. Therefore, we assume that these may be subject only to bounds (the DMs would fix the values for bounds $q_{j}^{1}, q_{j}^{\mathrm{u}}$, etc.):

$q_{j} \in\left[q_{j}^{1}, q_{j}^{\mathrm{u}}\right], \quad j=1, \ldots, n$

and

$p_{j} \in\left[p_{j}^{1}, p_{j}^{\mathrm{u}}\right], \quad j=1, \ldots, n$.

As mentioned in the previous section, additional constraints may be needed to ensure

$v_{j} \geq p_{j} \geq q_{j}, \quad j=1, \ldots, n$.
Concerning the performances of the actions, we consider that there may be constraints involving actions other than $a_{1}$ and $a_{2}$. For each criterion (let $j$ denote its index), DMs may define a polytope $G_{j}$ of admissible performances for $m$ actions. This polytope is defined by constraints such as $g_{j}\left(a_{x}\right) \geq 3 g_{j}\left(a_{y}\right), \quad g_{j}\left(a_{x}\right) \geq g_{j}\left(a_{y}\right)+2 \quad$ or $\quad g_{j}\left(a_{x}\right)+$ $g_{j}\left(a_{y}\right)=2 g_{j}\left(a_{z}\right)$. The resulting constraints can be represented as:

$$
\begin{aligned}
& \left(g_{j}\left(a_{1}\right), g_{j}\left(a_{2}\right), \ldots, g_{j}\left(a_{m}\right)\right) \in G_{j} \subset \mathbb{R}^{m}, \\
& \quad j=1, \ldots, n .
\end{aligned}
$$

Concerning importance coefficients, we assume that DMs define a polytope $K$ through linear constraints of the type $k_{i} \geq \alpha k_{j}, L_{j} \geq k_{i} \geq l_{j}, \alpha_{a} k_{a}+$ $\alpha_{b} k_{b}+\cdots+\alpha_{c} k_{c} \geq 0$, or $k_{1} / k_{2}+\cdots+k_{n}=1$. These constraints can be represented as:

$\left(k_{1}, \ldots, k_{n}\right) \in K \subset \mathbb{R}^{n}$.

Finally, let us consider the veto thresholds, which also carry information concerning how important each criterion is compared to others. For this reason, the veto threshold value for one criterion may be constrained by veto threshold values for other criteria. We consider three different situations:

Type (1) (bounds only)

$v_{j} \in\left[v_{j}^{1}, v_{j}^{\mathrm{u}}\right], \quad j=1, \ldots, n$;

Type (2) (polytope)

$\left(v_{1}, \ldots v_{n}\right) \in V \subset \mathbb{R}^{n}$;

Type (3) (dependence on $k_{j}$ )

$v_{j}=p_{j}+\alpha_{j} / k_{j}$, with $\alpha_{j} \in\left[\alpha_{j}^{1}, \alpha_{j}^{\mathrm{u}}\right], \quad j=1, \ldots, n$.

We assume that all parameters are non-negative and $G_{j}(j=1, \ldots, n), K$ and $V$ are closed and bounded. Hence, $T$ is a polytope. Mousseau (1993) has devised a questioning protocol and an interactive computer program to infer (4.2), (4.3), (4.6) and (4.7a) from the DMs' answers.

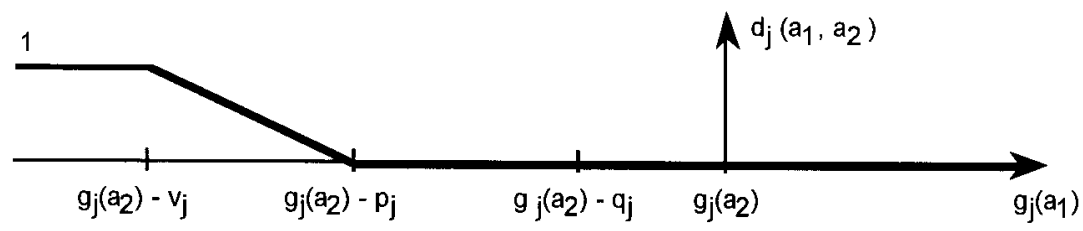

Figure 3. Single-criterion discordance index. 


\section{COMPUTING THE VARIATION INTERVAL OF A CREDIBILITY INDEX}

\subsection{Maximization of the credibility index}

Given any pair of actions $\left(a_{1}, a_{2}\right)$, we discuss in this section how to find the maximum $\sigma\left(a_{1}, a_{2}, t\right)$, subject to $t \in T$. We will refer to Propositions $1-8$, which are presented in the Appendix. The interested reader may read the Appendix before reading this and the next sections.

Consider a polytope $T$ as defined in the previous section. Some subsets of parameters may be varied independently of others: we can start by fixing the performances $g_{j}\left(a_{1}\right), \quad g_{j}\left(a_{2}\right) \quad(j=$ $1, \ldots, n)$, then the indifference and preference thresholds, and finally the importance coefficients and the veto thresholds. Since we wish to maximize $\sigma\left(a_{1}, a_{2}, t\right)$ we will always set these parameters to the values that most benefit action $a_{1}$. We suggest to proceed as follows:

Let $J=\{1, \ldots, n\}$ denote the set of criteria indices;

Let $J^{d}=\left\{1, \ldots, n: \Delta_{j}<0\right\}$ denote the subset of criteria indices where $a_{1}$ is worse than $a_{2}$.

1. The performances of the actions are the first parameters to be fixed. For each criterion, this amounts to solve a linear program to maximize $\Delta_{j}$ (see Proposition 1):

$$
\begin{aligned}
& \left(g_{j}\left(a_{1}\right), g_{j}\left(a_{2}\right)\right) \\
& \leftarrow \arg \max \left\{\Delta_{j}:\left(g_{j}\left(a_{1}\right), \ldots, g_{j}\left(a_{m}\right)\right) \in G_{j}\right\}, \\
& \quad j=1, \ldots, n .
\end{aligned}
$$

2. Set the indifference and preference thresholds to their maximum values (see Proposition 1). Note that the thresholds $q_{j}$ and $p_{j}$ affect the credibility index only if $j \in J^{\mathrm{d}}$. We assume that bounds are such that the constraints $v_{j} \geq p_{j} \geq$ $q_{j}(j=1, \ldots, n)$ are satisfied:

$q_{j} \leftarrow q_{j}^{\mathrm{u}}$ and $p_{j} \leftarrow p_{j}^{\mathrm{u}}, j \in J^{\mathrm{d}}$

(for $j \notin J^{\mathrm{d}}$, let $q_{j}$ and $p_{j}$ take any admissible values).

3. Type 1 constraint (4.7a). In this case the veto thresholds do not interact with each other nor with any other parameter. Hence, they can be fixed to their maximum values (see Proposition 1):

$v_{j} \leftarrow v_{j}^{\mathrm{u}}, j \in J^{\mathrm{d}}$

(for $j \notin J^{\mathrm{d}}$, let $v_{j}$ take any admissible value).
Now, only the importance coefficients remain to be fixed, constrained by (4.6). To find the maximum credibility $\sigma\left(a_{1}, a_{2}\right)$ we only have to find the maximum concordance $c\left(a_{1}, a_{2}\right)$. This amounts to solve a linear program, since $K$ is a polytope and $c\left(a_{1}, a_{2}, k\right)$ is linear (Proposition 2).

$$
\begin{aligned}
& \left(k_{1}, \ldots, k_{n}\right) \\
& \leftarrow \arg \max \left\{c\left(a_{1}, a_{2}, k\right):\left(k_{1}, \ldots, k_{n}\right) \in K\right\} .
\end{aligned}
$$

(PMax1)

Type 2 constraint (4.7b). In this case the veto thresholds are interdependent, although independent from the importance coefficients. Therefore, we may separately optimize the two sets of parameters. First, solve the linear program (PMax1) to maximize $c\left(a_{1}, a_{2}\right)$. If the optimum value of (PMax1) is zero or one, then stop (the maximum $\sigma\left(a_{1}, a_{2}\right)$ is zero or one, respectively). Otherwise, take the optimum $c\left(a_{1}, a_{2}\right)$ found and maximize $\sigma\left(a_{1}, a_{2}, v\right)$. To prevent $\sigma\left(a_{1}, a_{2}, v\right)$ from being null, add the following (bound) constraints to the polytope $V$ :

$v_{j} \geq-\Delta_{j}+\varepsilon$,

$j \in J^{\mathrm{d}}$ (where $\varepsilon$ is a small positive number).

If the set of constraints becomes inconsistent, then stop (the maximum $\sigma\left(a_{1}, a_{2}\right)$ is 0 ). Otherwise, maximize the credibility as a function of the veto thresholds:

$\left(v_{1}, \ldots, v_{n}\right)$

$\leftarrow \arg \max \left\{\sigma\left(a_{1}, a_{2}, v\right):\left(v_{1}, \ldots, v_{n}\right) \in V\right.$,

$\left.v_{j} \geq-\Delta_{j}+\varepsilon\left(j \in J^{\mathrm{d}}\right)\right\}$.

(PMax2)

By Proposition 4, $\sigma(v)$ is strictly quasiconcave under these conditions. As a consequence, the local maximum of this nonlinear program must be a global maximizer (e.g. see Bazaraa et al., 1993). An alternative approach is suggested by Proposition 5 .

Type 3 constraint (4.7c). In this case the veto thresholds are determined by the importance and $\alpha_{j}$ coefficients. The latter are subject only to bounds and may therefore be fixed to the values that makes the veto thresholds $v_{j}=p_{j}+$ $\alpha_{j} / k_{j}$ as high as possible (Proposition 1):

$\alpha_{j} \rightarrow \alpha_{j}^{\mathrm{u}}, j \in J^{\mathrm{d}}$

(for $j \notin J^{\mathrm{d}}$, let $\alpha_{j}$ take any admissible value). 
To prevent $\sigma\left(a_{1}, a_{2}\right)$ from being null, add the following (bound) constraints to $K$ :

$k_{j} \leq \alpha_{j} /\left(-\Delta_{j}-p_{j}\right)-\varepsilon, j \in J^{\mathrm{d}}$

(where $\varepsilon$ is a small positive number).

If the set of constraints becomes inconsistent, then stop (the maximum $\sigma\left(a_{1}, a_{2}\right)$ is 0 ). Otherwise, maximize the credibility as a function of the importance coefficients:

$$
\begin{aligned}
& \left(k_{1}, \ldots, k_{n}\right) \\
& \leftarrow \arg \max \left\{\sigma\left(a_{1}, a_{2}, k\right):\left(k_{1}, \ldots k_{n}\right) \in K,\right. \\
& \left.k_{j} \leq \alpha_{j} /\left(-\Delta_{j}-p_{j}\right)-\varepsilon\left(j \in J^{\mathrm{d}}\right)\right\} .
\end{aligned}
$$

By Propositon $9, \sigma(k)$ is strictly quasiconcave, since at this moment $0<\sigma(k)<1$. Thus, any local maximum of this nonlinear program must be a global maximizer. Alternatively, according to our experience, it is a good idea to maximize concordance at first. If the solution $k^{*}$ with optimum concordance is not weakened by discordance at any criteria (i.e. $\left.\mathrm{c}\left(k^{*}\right)=\sigma\left(k^{*}\right)\right)$, then the optimum credibility will have been found. Otherwise, proceed as described above.

We have shown that maximizing $\left\{\sigma\left(a_{1}, a_{2}, t\right)\right.$ : $t \in T\}$ may be divided into simpler problems. Problems (PMax0) and (PMax1) can be solved by linear programming techniques, but nonlinear programs (PMax2) and (PMax3) are generally more difficult to solve. Fortunately, the search for the optimum is simplified to the extent that the constraints are linear and there is only a local (hence global) maximum. However, this search must account for the nondifferentiability of the objective function. In Lemaréchal (1989) and references contained therein the reader may find many methods to cope with nonsmooth problems like these, that use generalized notions of derivatives and gradients. Most of these consider the problem of minimizing a convex function, or, equivalently, maximizing a concave function, which often may be extended to address quasiconcavity (see as an example Gromicho, 1998).

\subsection{Minimization of the credibility index}

We now discuss how to minimize $\sigma\left(a_{1}, a_{2}, t\right)$, subject to $t \in T$, given any pair of actions $\left(a_{1}, a_{2}\right)$. We will divide the problem into subproblems as in the previous section. Since we wish to minimize $\sigma\left(a_{1}, a_{2}, t\right)$, we will always set the parameters to the values that least benefit action $a_{1}$. We suggest to proceed as follows:

Let $J=\{1, \ldots, n\}$ denote the set of criteria indices;

Let $J^{\mathrm{d}}=\left\{1, \ldots, n: \Delta_{j}<0\right\}$ denote the subset of criteria indices where $a_{1}$ is worse than $a_{2}$.

1. The performances of the actions are the first parameters to be fixed. For each criterion, this amounts to solve a linear program to minimize $\Delta_{j}$ (see Proposition 1):

$$
\begin{aligned}
& \left(g_{j}\left(a_{1}\right), g_{j}\left(a_{2}\right)\right) \\
& \leftarrow \arg \min \left\{\Delta_{j}:\left(g_{j}\left(a_{1}\right), \ldots, g_{j}\left(a_{m}\right)\right) \in G_{j}\right\}, \\
& \quad j=1, \ldots, n .
\end{aligned}
$$

2. Set the indifference and preference thresholds to their minimum values (see Proposition 1). Once more, these thresholds affect the credibility index only for the criteria where $a_{1}$ is worse than $a_{2}$. We assume that bounds are such that the constraints $v_{j} \geq p_{j} \geq q_{j} \quad(j=$ $1, \ldots, n)$ are satisfied:

$q_{j} \leftarrow q_{j}^{1}$ and $p_{j} \leftarrow p_{j}^{1}, j \in J^{\mathrm{d}}$

(for $j \notin J^{\mathrm{d}}$, let $q_{j}$ and $p_{j}$ take any admissible values).

3. Type 1 constraint (4.7a). In this case the veto thresholds do not interact with each other nor with any other parameter. Hence, they can be fixed to their minimum values (see Proposition 1):

$v_{j} \leftarrow v_{j}^{1}, j \in j^{\mathrm{d}}$

(for $j \notin J^{\mathrm{d}}$, let $v_{j}$ take any admissible value).

Now, only the importance coefficients remain to be fixed, constrained by (4.6). To find the minimum credibility $\sigma\left(a_{1}, a_{2}\right)$ we only have to find the minimum concordance $c\left(a_{1}, a_{2}\right)$ by solving the following linear program:

$\left(k_{1}, \ldots, k_{n}\right)$

$\leftarrow \arg \min \left\{c\left(a_{1}, a_{2}, k\right):\left(k_{1}, \ldots, k_{n}\right) \in K\right\}$.

(Pmin1)

Type 2 constraint (4.7b). In this case the veto thresholds are interdependent, although independent from the importance coefficients. First, test whether any veto occurs, i.e.:

$\exists\left(v_{1}, \ldots, v_{n}\right) \in V, j \in J^{\mathrm{d}}: \quad v_{j}<-\Delta_{j}$ ?

If true, then the minimum credibility is zero. Otherwise, proceed as in the maximization case. First, solve the linear program (Pmin1) 
Table I. Performances (to maximize, hence the negative values) and fixed thresholds

\begin{tabular}{rllllllllll}
\hline & $\begin{array}{l}\text { Rent } \\
\left(g_{1}\right)\end{array}$ & $\begin{array}{l}\text { Sqr mt } \\
\left(g_{2}\right)\end{array}$ & $\begin{array}{l}\text { Work } \\
\left(g_{3}\right)\end{array}$ & $\begin{array}{l}\text { Rooms } \\
\left(g_{4}\right)\end{array}$ & $\begin{array}{l}\text { Shops } \\
\left(g_{5}\right)\end{array}$ & $\begin{array}{l}\text { School } \\
\left(g_{6}\right)\end{array}$ & $\begin{array}{l}\text { Garden } \\
\left(g_{7}\right)\end{array}$ & $\begin{array}{l}\text { Caution } \\
\left(g_{8}\right)\end{array}$ & $\begin{array}{l}\text { Transp. } \\
\left(g_{9}\right)\end{array}$ & $\begin{array}{l}\text { Neighb. } \\
\left(g_{10}\right)\end{array}$ \\
\hline$a_{1}$ & -4950 & 64 & -43 & 4 & -580 & -500 & 540 & -5500 & 4 & 3 \\
$a_{2}$ & -4350 & 59 & -32 & 3 & -450 & -460 & 430 & -3750 & 1 & 2 \\
$q_{j}$ & 100 & 3 & 5 & 0.1 & 50 & 50 & 20 & 1000 & 0.4 & 0.4 \\
$p_{j}$ & 300 & 6 & 10 & 0.2 & 150 & 150 & 50 & 2000 & 0.5 & 0.5 \\
\hline
\end{tabular}

to find the minimum concordance. If its optimum value happens to be zero or one, then stop (the minimum credibility will be zero or one, respectively). Otherwise, minimize the credibility as a function of the veto thresholds:

$\left(v_{1}, \ldots, v_{n}\right)$

$\leftarrow \arg \min \left\{\sigma\left(a_{1}, a_{2}, v\right):\left(v_{1}, \ldots, v_{n}\right) \in V\right\}$.

(Pmin2)

By Proposition 4, $\sigma(v)$ is strictly quasiconcave. Therefore, since $V$ is a polytope, the global minimum of this nonlinear program lays on a vertex of $V$ (e.g. see Bazaraa et al., 1993, p. 107 , for a proof).

Type 3 constraint (4.7c). In this case the veto thresholds are determined by the importance and $\alpha_{j}$ coefficients. Fix the $\alpha_{j}$ to make $v_{j}=p_{j}+$ $\alpha_{j} / k_{j}$ as low as possible (Proposition 1):

$\alpha_{j} \leftarrow \alpha_{j}^{1}, j \in J^{\mathrm{d}}$

(for $j \notin J^{\mathrm{d}}$, let $\alpha_{j}$ take any admissible value).

Afterwards, test whether veto occurs for any criterion:

$\exists\left(k_{1}, \ldots, k_{n}\right) \in K, j \in J^{\mathrm{d}}: k_{j} \geq \alpha_{j} /\left(-\Delta_{j}-p_{j}\right) ?$

If true, then the minimum credibility is zero. Otherwise, minimize the credibility as a function of the importance coefficients:

$\left(k_{1}, \ldots, k_{n}\right)$

$\leftarrow \arg \min \left\{\sigma\left(a_{1}, a_{2}, k\right):\left(k_{1}, \ldots, k_{n}\right) \in K\right\}$.

(Pmin3)

By Proposition $4, \sigma(k)$ is strictly quasiconcave. Therefore, since $K$ is a polytope, the global minimum of this nonlinear program lays on a vertex of $K$.

As in the previous section, we have shown that minimizing $\left\{\sigma\left(a_{1}, a_{2}, t\right): t \in T\right\}$ may be divided into simpler problems. Problems (Pmin0) and (Pmin1) can be solved by linear programming techniques, but nonlinear programs (Pmin2) and (Pmin3) are more difficult to solve. Since the functions are quasiconcave, these are global optimization problems (Rinnooy Kan and Timmer, 1989), hence there may exist multiple local minima. Concave (or quasiconcave) minimization is a difficult problem for which many efficient methods have been proposed (Horst and Tuy, 1996). If the polytope does not have many vertices, or if it is repeatedly used for many problems with different pairs of actions, then an algorithm for enumerating all the vertices of the polytope (e.g. Avis and Fukuda, 1992) may be an appropriate choice.

\section{ILLUSTRATIVE EXAMPLES}

\subsection{Finding the variation range of a credibility index}

We first present a few numerical experiments to illustrate this approach in detail. Since it was out of the scope of our work to find the best algorithms or software available, we used programs that were easily available to us and to the reader. We have based our experiments on an example by Mousseau (1993), where ten criteria are used to evaluate apartments for rent. We have invented two actions, $a_{1}$ and $a_{2}$, with fixed performances (see Table I) in the ranges considered by Mousseau. Table I also presents values for the indifference and preference thresholds fixed by us. Following Mousseau (1993), we considered some constraints on the importance coefficients (which he inferred from the answers of a DM) defining a polytope $K$ :

$$
\begin{array}{llll}
k_{1}<k_{2} & k_{2}<2 k_{1} & & \\
k_{3}<k_{2} & k_{3}<k_{1}+k_{2} & & \\
k_{4}>k_{2}+k_{3}-k_{1} & k_{4}>k_{1}+k_{3} & k_{4}<k_{2}+k_{3} & \\
k_{5}>k_{4} & k_{5}<k_{2}+k_{3} & & \\
k_{6}>k_{2}+k_{3}-2 k_{1} & k_{6}>k_{5} & k_{6}<k_{2}+k_{3} & \\
k_{7}>k_{1}+k_{6} & k_{7}>k_{4}+k_{5}-k_{1} & k_{7}>k_{6} & k_{7}<k_{4}+k_{5}+k_{1}
\end{array}
$$


Table II. Middle values for the importance coefficients

\begin{tabular}{lllllllllll}
\hline & $k_{1}$ & $k_{2}$ & $k_{3}$ & $k_{4}$ & $k_{5}$ & $k_{6}$ & $k_{7}$ & $k_{8}$ & $k_{9}$ & $k_{10}$ \\
\hline Original & 1 & 1.5 & 2 & 3.25 & 3.38 & 3.44 & 6.63 & 7.78 & 3.38 & 3.44 \\
Normalized & 0.0279 & 0.0419 & 0.0559 & 0.0908 & 0.0944 & 0.0961 & 0.1852 & 0.2173 & 0.0944 & 0.0961 \\
\hline
\end{tabular}

$k_{8}>k_{5}+k_{6}-k_{1} \quad k_{8}>k_{4}+k_{6} \quad k_{8}>k_{7} \quad k_{8}<k_{4}+k_{7}-k_{1}$

$k_{9}=k_{5} \quad k_{10}=k_{6}$

$k_{1}, \ldots, k_{10} \geq 0$

Mousseau suggested using the 'middle' values after fixing, for instance, $k_{1}=1$ (Table II).

\subsubsection{First experiment}

We considered a situation with a Type 1 constraint with fixed veto thresholds $v=$ $(700,15,30,2,500,500,200,5000,3,3) . \quad$ The concordance and discordance figures corresponding to the ordered pair $\left(a_{1}, a_{2}\right)$ and the values in Table II were:

$c\left(a_{1}, a_{2}, k\right)=0.678 ; \sigma\left(a_{1}, a_{2}, k\right)=0.678$

(discordance not very strong).

Afterwards, we used Microsoft Excel's Solver to maximize and minimize $c\left(a_{1}, a_{2}, k\right)$ subject to $k \in K$. The variation range computed was the following:

$$
\begin{aligned}
0.653 & \leq c\left(a_{1}, a_{2}, k\right) \\
& \leq 0.704, \text { corresponding to } 0.653 \\
& \leq \sigma\left(a_{1}, a_{2}, k\right) \leq 0.704 .
\end{aligned}
$$

Although we could add a small perturbation to each inequality to force strictness, we have let the Solver consider the inequalities as not strict. The Solver's calculations were practically instantaneous on a standard Pentium PC (166 MHz).

\subsubsection{Second experiment}

We considered a situation with a Type 3 constraint, where $v_{j}=p_{j}+\alpha_{j} / k_{j}(j=1, \ldots, n)$ with the values for $\alpha_{j}$ fixed by us to be $\alpha=$ $(11.2,0.4,1.2,0.16,33,34,10,650,0.25,0.25)$.

Considering the middle values for the $k_{j}$ this corresponds to $v=(700.96,15.5467,31.48,1.9625$, $499.53,503.84,104,4991,3.1479,3.1017)$ and to the following results:

$c\left(a_{1}, a_{2}, k\right)=0.678 ; \sigma\left(a_{1}, a_{2}, k\right)=0.529$

(discordance is no longer negligible).
Using the spreadsheet we readily verified that the minimum of $\sigma\left(a_{1}, a_{2}, k\right)$ is zero. To maximize this credibility index we used the Solver again. This solver expects differentiable functions, but we had no other software that did not require this. For some initial values the solver stopped at a non-optimal solution. Experimenting with a few different initial values has led us to find a maximum of 0.703 (for a solution that does not lay on any vertex of the polytope $K)$. The calculations were practically instantaneous. The variation range of the credibility index was:

$0 \leq \sigma\left(a_{1}, a_{2}, k\right) \leq 0.703$.

As a remark, note that if we maximized and minimized $c\left(a_{1}, a_{2}, k\right)$ instead, we would obtain a misleading variation for $\sigma\left(a_{1}, a_{2}, k\right)$ :

$$
\begin{aligned}
& 0.653 \leq c\left(a_{1}, a_{2}, k\right) \leq 0.704, \text { corresponding to } \\
& 0.441 \leq \sigma\left(a_{1}, a_{2}, k\right) \leq 0.609 .
\end{aligned}
$$

\subsubsection{Third experiment}

We changed $\alpha_{1}$ to 16 and $\alpha_{3}$ to 1.2 , so that the minimum credibility would not be zero. The maximum credibility is now 0.704 (it corresponds to the vertex solution that maximizes concordance). Using David Avis' LRS implementation of the reverse search algorithm, available from its author, we found 46 vertices in less than a second. Then, we quickly found a vertex yielding a minimum credibility of 0.127 . Experimenting with Excel's solver, which stops when it finds any local minimum, we were lucky enough to find the global minimum at our first trial. The variation range of the credibility index was now:

$0.127 \leq \sigma\left(a_{1}, a_{2}, k\right) \leq 0.704$.

Notice that the result corresponding to the 'middle' values in Table II is $\sigma\left(a_{1}, a_{2}, k\right)=0.678$, which is far from the middle of the variation range. 
Table III. Evaluation table of five projects

\begin{tabular}{llcclll}
\hline Project & $\begin{array}{l}\text { Population } \\
\text { served } \\
\left(g_{1}: \max \right)\end{array}$ & $\begin{array}{l}\text { Incoming } \\
\text { traffic/km } \\
\left(g_{2}: \max \right)\end{array}$ & $\begin{array}{l}\text { Cost } / \mathrm{km} \\
\left(g_{3}: \min \right)\end{array}$ & $\begin{array}{l}\text { Internal rate } \\
\text { of return } \\
\left(g_{4}: \max \right)\end{array}$ & $\begin{array}{l}\text { Network } \\
\text { organization } \\
\left(g_{5}: \text { min }\right)\end{array}$ & $\begin{array}{l}\text { Effect over } \\
\text { urbanism } \\
\left(g_{6}: \min \right)\end{array}$ \\
\hline 5 & 12500 & 7100 & 110 & 4.6 & 2 & 2 \\
$7 \mathrm{~N}$ & 33100 & 11500 & 140 & 14.1 & 3 & 11 \\
$7 \mathrm{~S}$ & 24000 & 11200 & 160 & 12.0 & 12 & 7 \\
8 & 17100 & 4000 & 40 & 11.8 & 18 & 3 \\
$13 \mathrm{~B}$ & 37650 & 10400 & 130 & 12.2 & 3 & 12 \\
\hline
\end{tabular}

\subsection{Finding robust conclusions concerning top-ranked actions}

As an example on how this approach might be used, let us consider a case study by Roy and Hugonnard in the early eighties (see Roy and Bouyssou, 1993, Chapter 10), concerning the evaluation of projects for expanding the Paris Metro (subway) network. This study used ELECTRE IV to rank the projects by order of priority. This method was chosen because of the unavailability of information concerning the relative importance of the criteria. It has been developed for that particular case study and, according to its authors, may be used when there is a need to progress without setting importance coefficients, as long as no criterion weighs more than a coalition of half of the criteria, and no criterion weighs so little that it could be removed.

The original study used six criteria and 12 projects. In this example we consider the five projects ranked at the topmost levels (Table III). The original study used two sets of values for the indifference and preference thresholds, which we consider here to be lower and upper bounds (Table IV). Following the original study, we considered $v_{j}=2 p_{j}$.

In this example, we consider variable values (partial information) concerning the importance coefficients for the six criteria. If all criteria had the same weight, then $k_{j}=1 / 6(j=1, \ldots, 6)$. We chose to let these parameters take different values, constrained by:

$\left(k_{1}, \ldots, k_{6}\right) \in K=\left\{\left(k_{1}, \ldots, k_{6}\right)\right.$ :

$1 / 2 \leq k_{j} \leq 3 / 12(j=1, \ldots, 6)$ and

$\left.k_{1}+k_{2}+\cdots+k_{6}=1\right\}$.

These constraints were chosen so that one criterion could not weigh more than a coalition of three other criteria. The last constraint was included to comply with (3.2).
Allowing the importance coefficients to vary within $K$, the indifference thresholds to vary within $q_{j} \in\left[q_{j}^{1}, q_{j}^{\mathrm{u}}\right]$, the preference thresholds to vary within $p_{j} \in\left[p_{j}^{1}, p_{j}^{\mathrm{u}}\right]$ and the veto thresholds to vary within $v_{j} \in\left[2 p_{j}^{1}, 2 p_{j}^{\mathrm{u}}\right]$ we find ourselves in a Type 1 situation (4.7a). To find the maximum (minimum) credibility we first fixed the thresholds to their upper (lower) bound and then maximized (minimized) the concordance using linear programming. We needed to solve 40 problems to find the results presented in Table V. If we consider that an outranking occurs if its credibility exceeds 0.7 (for instance), then we would conclude that:

- $7 \mathrm{~N}$ and 13B are indifferent (they outrank each other)', ' $13 \mathrm{~B}$ is preferred to $7 \mathrm{~S}$ (outranks but is not outranked)', '7N outranks $7 \mathrm{~S}$ ', '5 and 8 are incomparable (they do not outrank each other)', '7S and 8 are incomparable', '13B and 8 are incomparable' and ' $7 \mathrm{~N}$ and 8 are incomparable' are all robust conclusions. Even though the approaches are not comparable, we noticed that all these conclusions agree with the original study's, except the last two.

- '7N outranks 5', '7S outranks 5', '7S outranks $7 \mathrm{~N}$ ' and ' $13 \mathrm{~B}$ outranks 5' are the conclusions that are more affected by the fact that information is partial.

Table IV. Bounds for the indifference and preference thresholds

\begin{tabular}{lllllll}
\hline & $g_{1}$ & $g_{2}$ & $g_{3}$ & $g_{4}$ & $g_{5}$ & $g_{6}$ \\
\hline$q_{j}^{1}$ & $0.10 g_{1}$ & $0.15 g_{2}$ & $0.10 g_{3}$ & 1.0 & 2 & 2 \\
$p_{j}^{1}$ & $0.20 g_{1}$ & $0.30 g_{2}$ & $0.25 g_{3}$ & 2.5 & 5 & 5 \\
$q_{j}^{\mathrm{u}}$ & $0.15 g_{1}$ & $0.20 g_{2}$ & $0.15 g_{3}$ & 1.5 & 3 & 3 \\
$p_{j}^{\mathrm{u}}$ & $0.25 g_{1}$ & $0.35 g_{2}$ & $0.30 g_{3}$ & 3.5 & 6 & 6 \\
\hline
\end{tabular}


Table V. Pairwise comparison table of credibility indices under partial information

\begin{tabular}{llllll}
\hline & 5 & $7 \mathrm{~N}$ & $7 \mathrm{~S}$ & 8 & $13 \mathrm{~B}$ \\
\hline 5 & - & {$[0,0]$} & {$[0,0]$} & {$[0,0]$} & {$[0,0]$} \\
$7 \mathrm{~N}$ & {$[0.254,0.881]$} & - & {$[0.833,0.972]$} & {$[0,0]$} & {$[0.906,1]$} \\
$7 \mathrm{~S}$ & {$[0,0.778]$} & {$[0.013,0.808]$} & - & {$[0,0]$} & {$[0,0]$} \\
8 & {$[0,0]$} & {$[0,0]$} & {$[0,0]$} & - & {$[0,0]$} \\
$13 \mathrm{~B}$ & {$[0,0.915]$} & {$[0.85,0.983]$} & {$[0.750,0.944]$} & {$[0,0]$} & - \\
\hline
\end{tabular}

We would conclude that: $7 \mathrm{~N}$ and $13 \mathrm{~B}$ are indifferent; 7S can be excluded from the top position (because of 13B); and 8 is incomparable to the remaining projects. We would need more information (i.e. more constraints on $K$ and/or tighter bounds for the thresholds) to know how project 5 compares to the remaining projects.

\subsection{Finding robust conclusions concerning acceptance/rejection decisions}

Let us now exemplify how can the range of variation for the credibility indices be applied to accept/reject decisions. Hurson and Zopounidis (1997) have used ELECTRE TRI to evaluate 20 stocks from the Athens Stock Exchange (commercial sector) using six criteria (Table VI). When using the pessimistic variant of that method, the stocks were compared to two reference actions (Table VII). Consider a cut threshold $\lambda$. Each stock $a_{i}(i=1, \ldots, 20)$ is sorted into category 'Accept' if $\sigma\left(a_{i}, b^{\mathrm{h}}\right) \geq \lambda$; it is sorted into category 'Unknown' if $\sigma\left(a_{i}, b^{\mathrm{h}}\right)<\lambda$, but $\sigma\left(a_{i}, b^{l}\right) \geq \lambda$; it is sorted into category 'Reject' if $\sigma\left(a_{i}, b^{1}\right)<\lambda$ (which also implies $\left.\sigma\left(a_{i}, b^{\mathrm{h}}\right)<\lambda\right)$.

Consider now a framework of partial information, where there is a single reference action $b^{\mathrm{r}}$ with a variable performance profile: $g_{j}\left(b^{\mathrm{r}}\right) \in\left[g_{j}\left(b^{1}\right)\right.$, $\left.g_{j}\left(b^{\mathrm{h}}\right)\right], j=1, \ldots, 6$ (bounds in Table VII). Then, consider an approach that sorts stock $a_{i}$ into 'Accept' if min $\sigma\left(a_{i}, b^{\mathrm{r}}\right) \geq \lambda$; into 'Reject' if $\max$ $\sigma\left(a_{i}, b^{\mathrm{r}}\right)<\lambda$; into 'Unknown' otherwise. Not only this approach is equivalent to the previous one, but it can be extended to consider more parameters subject to partial information.

We consider that the values of Hurson and Zopounidis (1997) for the indifference and preference thresholds are fixed and consider that the values they provided for the reference actions (Table VII) are bounds. Since they do not provide information on the importance coefficients, let us suppose:

$$
\begin{aligned}
& \left(k_{1}, \ldots, k_{6}\right) \in K=\left\{\left(k_{1}, \ldots, k_{6}\right):\right. \\
& 1 / 12 \leq k_{j} \leq 3 / 12(j=1, \ldots, 6) \text { and } \\
& \left.k_{1}+k_{2}+\cdots+k_{6}=1\right\} .
\end{aligned}
$$

Finally, let us imagine a Type 3 situation $(4.7 \mathrm{c})$, where the veto thresholds are not fixed: $v_{j}=p_{j}+$ $\alpha_{j} / k_{j}$, with $\alpha_{j}$ such that the veto values coincide with Table VII when $k_{j}=1 / 6(=1, \ldots, 6)$.

Using the software referred to in the section 'Finding the Variation Range of a Credibility Index', all stocks were assigned to category 'Unknown' (considering $\lambda=0.5$ ). As a second experiment, we added the kind of constraints that would appear if the DMs provided a weak order on the importance of the criteria:

$k_{5} \geq k_{4} \geq k_{1} \geq k_{2} \geq k_{3} \geq k_{6}$.

The results for the two experiments are depicted in Table VIII. As more information is added, fewer stocks are sorted into category 'Unknown'. As a note, for most of the maximization problems we did not need to use nonlinear programming, because the solution with maximum concordance (obtained by linear programming) was also optimal in terms of credibility.

\section{SUMMARY AND FUTURE RESEARCH}

We have discussed the potential advantages of robustness analysis to cope with partial information on the values of parameters for a decision aid method. In this context, we considered Roy's (1997) definition of a robust conclusion and introduced the notions of absolute, (relative) binary and (relative) unary robust conclusions. We then briefly reviewed some approaches to cope with partial information, showing the role that optimization can play.

This work has then focused on the first phase of the ELECTRE methods that build fuzzy out- 
Table VI. Evaluation table of 20 stocks from the commercial sector

\begin{tabular}{|c|c|c|c|c|c|c|}
\hline Stocks & $\begin{array}{l}\text { Return } \\
\left(g_{1}: \max \right)\end{array}$ & $\begin{array}{l}\text { Marketability } \\
\left(g_{2}: \max \right)\end{array}$ & $\begin{array}{l}\text { Beta } \\
\left(g_{3}: \min \right)\end{array}$ & $\begin{array}{l}\text { Earnings/price } \\
\left(g_{4}: \max \right)\end{array}$ & $\begin{array}{l}\text { Growth of } \\
\text { dividend } \\
\left(g_{5}: \max \right)\end{array}$ & $\begin{array}{l}\text { 'Acid test' } \\
\left(g_{6}: \max \right)\end{array}$ \\
\hline 1 & 0.82 & 0.45 & 0.26 & -4.7 & -100 & 0.45 \\
\hline 2 & 0.41 & 0.63 & 0.03 & 2.28 & -20 & 2.04 \\
\hline 3 & 0.57 & 0.20 & 0.10 & 6.08 & -33 & 1.08 \\
\hline 4 & 0.24 & 0.02 & 0.08 & 2.41 & -54 & 0.62 \\
\hline 5 & 0 & 0.46 & 0.62 & 5.04 & -77 & 3.02 \\
\hline 6 & 0.93 & 0.02 & 0.14 & 2.82 & 6.38 & 0.72 \\
\hline 7 & 0.01 & 0.69 & 0.77 & 7.55 & -40 & 3.23 \\
\hline 8 & 0.86 & 0.86 & 0.86 & 4.28 & 3.71 & 0.57 \\
\hline 9 & 2.16 & 0.60 & 0.12 & 2.11 & 56.3 & 0.51 \\
\hline 10 & 1.24 & 0.12 & 0.62 & 11.7 & 12.5 & 1.17 \\
\hline 11 & 0.80 & 0.58 & 0.62 & 13.7 & 34.6 & 1.54 \\
\hline 12 & 1.23 & 0.37 & 0.64 & 8.97 & 45.9 & 0.96 \\
\hline 13 & 0.24 & 0.28 & 0.73 & -1.8 & 0 & 0.72 \\
\hline 14 & 0.26 & 0.65 & 0.58 & 4.88 & 7.14 & 0.90 \\
\hline 15 & 1.10 & 0.76 & 0.54 & 0.29 & 0 & 0.73 \\
\hline 16 & 1.79 & 0.55 & 0.73 & 5.88 & -100 & 2.69 \\
\hline 17 & 1.02 & 1.06 & 0.82 & 5.5 & 6.38 & 0.73 \\
\hline 18 & 1.32 & 1.12 & 0.94 & 12.1 & -61 & 2.69 \\
\hline 19 & 1.36 & 0.04 & 1.02 & 1.79 & 110 & 2.31 \\
\hline 20 & 0.57 & 0.17 & 0.23 & -12 & 0 & 0.52 \\
\hline
\end{tabular}

ranking relations. We studied the problem of finding the maximum and minimum credibility of an outranking given a pair of actions and linear constraints on the values of the parameters. Assuming a reasonable set of constraints, where some of the parameters may vary independently from some others, we have proved some characteristics of the nonlinear credibility function that may ease its maximization or minimization. On considering these optimization problems, we suggested how they may be solved.

Knowing the maximum and minimum credibility $\sigma\left(a_{1}, a_{2}\right)$ for the pair of actions $\left(a_{1}, a_{2}\right)$ and a range $\left[\lambda_{\min }, \lambda_{\max }\right]$ for the cut threshold $\lambda$, we can test the robustness of the relative binary premises ' $a_{1} S a_{2}$ ' and 'not $\left(a_{1} S a_{2}\right)$ '. The first is robust if $\min \left\{\sigma\left(a_{1}, a_{2}\right)\right\} \geq \lambda_{\max }$, while the latter is robust if $\max \left\{\sigma\left(a_{1}, a_{2}\right)\right\} \leq \lambda_{\min }$. If several actions are compared, then this approach lets the DMs know which indices are most affected by the fact that information is partial.

Future research may address the choice of the best algorithms to solve the optimization problems. As in other circumstances (Dias et al., 1997), parallel processing may play a positive role. A second stream of research is to find how the credibility function may be transformed to gain nicer properties, such as concavity instead of quasiconcavity. A third stream, which we deem more important, is how to build on this work to test the robustness of conclusions from the exploitation phase of ELECTRE methods. In this context, the analysis performed here is very important concerning the ELECTRE TRI method (Dias and Clímaco, 1998b). Some of the ideas put forward in this paper could also be useful concerning other decision aid methods, especially those based on outranking relations.

By demanding less information from the DMs, we are encouraging a faster (yet more confident) progress in a decision process, at the cost of providing intervals as results, instead of precise values. This in turn creates another stream for future research: to find exploitation techniques adequate to deal with these 'interval' relations.

Table VII. Reference actions and local thresholds

\begin{tabular}{|c|c|c|c|c|c|c|}
\hline & $g_{1}$ & $g_{2}$ & $g_{3}$ & $g_{4}$ & $g_{5}$ & $g_{6}$ \\
\hline$b^{\mathrm{h}}$ & 1 & 0.70 & 0.25 & 6 & 10 & 1.1 \\
\hline$b^{1}$ & 0.5 & 0.35 & 0.60 & 2.5 & 0 & 0.7 \\
\hline$q_{j}$ & 0.05 & 0.05 & 0 & 0.1 & 8.72 & 0.05 \\
\hline$p_{j}$ & 0.25 & 0.20 & 0.2 & 0.5 & 10 & 0.25 \\
\hline$v_{j}$ & 2 & 1 & 1 & 10 & 180 & 2.75 \\
\hline
\end{tabular}


Table VIII. Results of sorting under partial information

\begin{tabular}{|c|c|c|c|c|}
\hline & \multicolumn{2}{|c|}{ Range of credibility index } & \multicolumn{2}{|c|}{ Accept $/$ Reject $(\lambda=0.5)$} \\
\hline & $\begin{array}{l}\text { No ranking of } \\
\text { criteria }\end{array}$ & $\begin{array}{l}\text { Ranking of } \\
\text { criteria }\end{array}$ & $\begin{array}{l}\text { No ranking of } \\
\text { criteria }\end{array}$ & $\begin{array}{l}\text { Ranking of } \\
\text { criteria }\end{array}$ \\
\hline 1 & {$[0,0.75]$} & {$[0,0.277]$} & $?$ & Reject \\
\hline 2 & {$[0.144,0.875]$} & {$[0144,0.75]$} & $?$ & $?$ \\
\hline 3 & {$[0.137,0.861]$} & {$[0.318,0.722]$} & $?$ & $?$ \\
\hline 4 & {$[0.003,0.713]$} & {$[0.007,0.481]$} & $?$ & Reject \\
\hline 5 & {$[0.09,0.825]$} & {$[0.011,0.65]$} & $?$ & $?$ \\
\hline 6 & {$[0.024,0.917]$} & {$[0.23,0.917]$} & ? & $?$ \\
\hline 7 & {$[0.056,0.763]$} & {$[0.261,0.525]$} & ? & $?$ \\
\hline 8 & {$[0.062,0.883]$} & {$[0.36,0.883]$} & $?$ & $?$ \\
\hline 9 & {$[0.332,0.881]$} & {$[0.583,0.821]$} & ? & Accept \\
\hline 10 & {$[0.288,0.908]$} & {$[0.663,0.908]$} & $?$ & Accept \\
\hline 11 & {$[0.446,0.992]$} & {$[0.594,0.992]$} & $?$ & Accept \\
\hline 12 & {$[0.388,0.983]$} & {$[0.592,0.983]$} & ? & Accept \\
\hline 13 & {$[0,0.746]$} & {$[0,0.583]$} & ? & $?$ \\
\hline 14 & {$[0.125,0.921]$} & {$[0.318,0.881]$} & $?$ & $?$ \\
\hline 15 & {$[0.035,0.917]$} & {$[0.059,0.85]$} & $?$ & $?$ \\
\hline 16 & {$[0.041,0.863]$} & {$[0.102,0.738]$} & $?$ & $?$ \\
\hline 17 & {$[0.102,0.917]$} & {$[0.5,0.917]$} & ? & Accept \\
\hline 18 & {$[0.075,0.833]$} & {$[0.543,0.708]$} & ? & Accept \\
\hline 19 & {$[0,0.75]$} & {$[0.118,0.583]$} & $?$ & $?$ \\
\hline 20 & {$[0,0.79]$} & {$[0,0]$} & $?$ & Reject \\
\hline
\end{tabular}

\section{ACKNOWLEDGEMENTS}

This research has been partially supported by PRAXIS XXI Technological Development and Scientific Research contracts PCSH/CEG/0028/96 and $2 / 2.1 / \mathrm{MAT} / 465 / 94$. We thank David Avis for making his software implementing LRS publicly available at the ftp site mutt.cs.mcgill.ca, at the directory $\mathrm{pub} / \mathrm{C}$.

\section{APPENDIX A}

This appendix presents some results concerning the credibility index function $\sigma\left(a_{1}, a_{2}, t\right)$, where $t$ is a combination of parameter values in a given polytope $T$. We assume that the reader has already read the sections 'Computing ELECTRE's Credibility Index' and 'Constraints on the Parameter Values' and use the same notation. Throughout this appendix, we will consider an ordered pair of actions $\left(a_{1}, a_{2}\right)$. Hence, we will sometimes refer to the credibility function as $\sigma(t): T \rightarrow[0,1]$, omitting the reference to the identity of the actions. The function

$$
\begin{aligned}
\sigma(t) & \equiv \sigma\left(a_{1}, a_{2}, t\right) \\
& =c\left(a_{1}, a_{2}, t\right) \prod_{\substack{j \in\{1, \ldots, n\}: \\
d_{j}\left(a_{1}, a_{2}, t\right)>c\left(a_{1}, a_{2}, t\right)}} \frac{1-d_{j}\left(a_{1}, a_{2}, t\right)}{1-c\left(a_{1}, a_{2}, t\right)}
\end{aligned}
$$

is obviously continuous but it has no derivative in a finite number of points of $T$, since neither $c($.) nor $d_{j}($.$) have. It increases when any c_{j}(t)$ increases and/or any $d_{j}(t)$ decreases, hence:

\section{Proposition 1}

If any $\Delta_{j}, q_{j}, p_{j}$ or $v_{j}$ increases $(j \in\{1, \ldots, n\})$, then $\sigma\left(a_{1}, a_{2}\right)$ does not decrease.

We omit the proof, since it is obvious given (3.1)-(3.4) (see also Figures 4 and 5).

Consider the polytope $K$ defined by (4.6). The next proposition states that the concordance index $c\left(a_{1}, a_{2}\right) \equiv c\left(a_{1}, a_{2}, k\right) \equiv c(k): K \rightarrow[0,1]$ is linear on $k$ when some parameters are fixed.

\section{Proposition 2}

Given fixed values for $q_{j}, p_{j}$ and $\Delta_{j}(j=1, \ldots, n)$, 
$\forall x, y \in K, \lambda \in[0,1], c((1-\lambda) x+\lambda y)$

$=(1-\lambda) c(x)+\lambda c(y)$.

We omit the proof, since it follows easily from (3.1) and (3.2).

Next we consider the cases when the veto thresholds cannot vary independently, i.e. constraints of Type 2 and Type 3 .

\section{A.1. Type 2 constraint}

Let us consider that all parameters are fixed, except the veto thresholds, constrained by $\left(v_{1}, \ldots, v_{n}\right)=v \in V$ (Type 2). If $c\left(a_{1}, a_{2}\right)=1$, then the credibility index equals one. Note also that if any veto occurs, then the credibility index equals zero. Therefore, we can restrict the ourselves to the case where the value of the credibility index is unknown (depends on $v \in V$ ), i.e. $c\left(a_{1}, a_{2}\right)<1$ and $v_{j} \geq-\Delta_{j}(j=1, \ldots, n)$. We will show that under these conditions the credibility index is a strictly quasiconcave function of $v$. Considering this domain let us write:

$$
\begin{aligned}
\sigma(v) & \equiv \sigma\left(a_{1}, a_{2}, v\right) \\
& =c\left(a_{1}, a_{2}\right) \prod_{\substack{j \in\{1, \ldots, n\}: \\
d_{j}\left(a_{1}, a_{2}, t\right)>c\left(a_{1}, a_{2}\right)}} \frac{1-d_{j}\left(a_{1}, a_{2}, v_{j}\right)}{1-c\left(a_{1}, a_{2}\right)} \\
& =c\left(a_{1}, a_{2}\right) \prod_{j=1}^{n} F D_{j}\left(v_{j}\right),
\end{aligned}
$$

where

$$
\begin{aligned}
F D_{j}\left(v_{j}\right) & \equiv F D_{j}\left(a_{1}, a_{2}, v_{j}\right) \\
& =\min \left\{1, \frac{1-d_{j}\left(a_{1}, a_{2}, v_{j}\right)}{1-c\left(a_{1}, a_{2}\right)}\right\} .
\end{aligned}
$$

The expression of $F D_{j}\left(v_{j}\right)$ is: otherwise

\section{Proposition 3}

$F D_{j}\left(v_{j}\right)$ is concave for $v_{j}>-\Delta_{j}$, i.e. $F D_{j}((1-$ $\lambda) x+\lambda y) \geq(1-\lambda) F D_{j}(x)+\lambda F D_{j}(y), \forall \lambda \in[0,1]$.

\section{Proof}

If $\Delta_{j} \geq-p_{j}$ then $\forall v_{j}, F D_{j}\left(v_{j}\right)=1$ and the proposition is true. Otherwise, $\Delta_{j}<-p_{j}$, hence:

$F D_{j}\left(v_{j}\right)=\min \left\{1, f_{2}\left(v_{j}\right)\right\}$,

where

$f_{2}\left(v_{j}\right)=\left(1-\frac{-\Delta_{j}-p_{j}}{v_{j}-p_{j}}\right) /\left(1-c\left(a_{1}, a_{2}\right)\right)$.

Since

$\frac{\mathrm{d}^{2} f_{2}}{\mathrm{~d} v_{j}^{2}}\left(v_{j}\right)=\frac{2\left(\Delta_{j}+p_{j}\right)}{1-c\left(a_{1}, a_{2}\right)} \cdot \frac{1}{\left(v_{j}-p_{j}\right)^{3}}<0$,

$f_{2}\left(v_{j}\right)$ is concave. $F D_{j}\left(v_{j}\right)$ is in this case the minimum between a constant and a concave function, therefore it is also concave.

\section{Proposition 4}

$\sigma(v)$ is strictly quasiconcave in $U:=\left\{v \in \mathbb{R}^{n}: v_{j}>\right.$ $\left.-\Delta_{j} \quad(j=1, \ldots, n)\right\}$, i.e. $\left.\forall \lambda \in\right] 0,1[, \quad x, y \in U$, $\sigma(x) \neq \sigma(y) \Rightarrow \sigma((1-\lambda) x+\lambda y)>\min \{\sigma(x), \sigma(y)\}$.

\section{Proof}

Let $\lambda \in] 0,1[$ and let $x, y \in U$, such that $\sigma(x) \neq$ $\sigma(y)$. Without loss of generality let $\sigma(x)<\sigma(y)$. Let us define:

$$
F D(v)=\prod_{j=1}^{n} F D_{j}\left(v_{j}\right)
$$

$F D_{j}\left(v_{j}\right)=\left\{\begin{array}{l}\left(1-\frac{-\Delta_{j}-p_{j}}{v_{j}-p_{j}}\right) /\left(1-c\left(a_{1}, a_{2}\right)\right), \quad \text { if } \Delta_{j}<-p_{j} \wedge v_{j}<p_{j}-\frac{p_{j}+\Delta_{j}}{c\left(a_{1}, a_{2}\right)} \\ 1,\end{array}\right.$

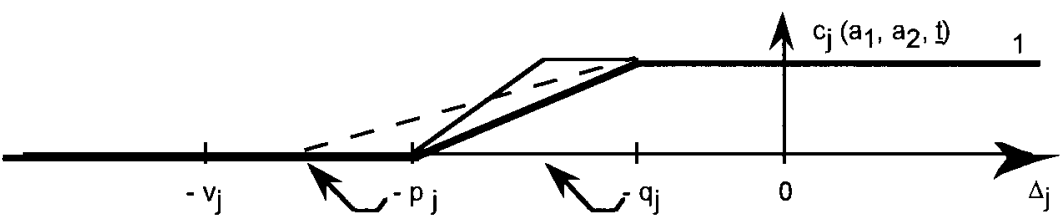

Figure 4. Single-criterion concordance index does not decrease. 


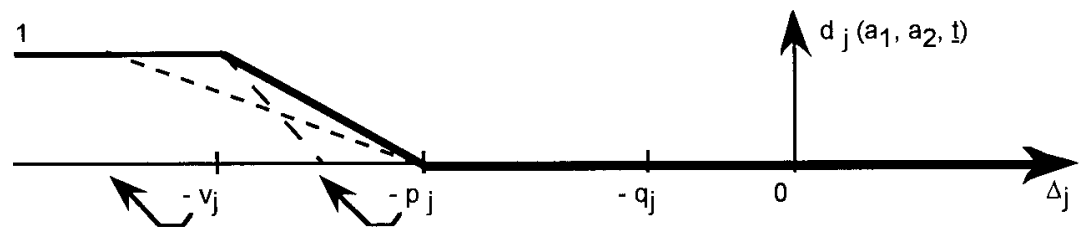

Figure 5. Single criterion discordance index does not increase.

Then, $\sigma(v)=c\left(a_{1}, a_{2}\right) F D(v)$. All parameters are fixed except the veto thresholds, hence $\sigma(x)<$ $\sigma(y) \Leftrightarrow F D(x)<F D(y)$. Since $U$ is convex, $F D((1-\lambda) x+\lambda y)>0$ and we may write:

$$
\begin{aligned}
\ln F D((1-\lambda) x+\lambda y) & =\ln \prod_{j=1}^{n} F D_{j}\left((1-\lambda) x_{j}+\lambda y_{j}\right) \\
& =\sum_{j=1}^{n} \ln F D_{j}\left((1-\lambda) x_{j}+\lambda y_{j}\right) .
\end{aligned}
$$

Since $F D_{j}($.$) (Proposition 3) and the logarithmic$ function are concave,

$$
\begin{aligned}
& \sum_{j=1}^{n} \ln F D_{j}\left((1-\lambda) x_{j}+\lambda y_{j}\right) \\
& \geq \sum_{j=1}^{n} \ln \left[(1-\lambda) F D_{j}\left(x_{j}\right)+\lambda F D_{j}\left(y_{j}\right)\right] \\
& \geq \sum_{j=1}^{n}\left[(1-\lambda) \ln F D_{j}\left(x_{j}\right)+\lambda \ln F D_{j}\left(y_{j}\right)\right] \\
& =(1-y) \sum_{j=1}^{n} \ln F D_{j}\left(x_{j}\right)+\lambda \sum_{j=1}^{n} \ln F D_{j}\left(y_{j}\right),
\end{aligned}
$$

i.e. $\ln F D((1-\lambda) x+\lambda y)$

$$
\geq(1-\lambda) \ln F D(x)+\lambda \ln F D(y) \text {. }
$$

Now, since $F D(x)<F D(y)$ and the logarithmic function is strictly increasing, we must have $\ln F D((1-\lambda) x+\lambda y)>(1-\lambda) \ln F D(x)+\lambda \ln$ $F D(x)=\ln F D(x)$. Therefore, $F D((1-\lambda) x+$ $\lambda y)>F D(x)$, which multiplied by $c\left(a_{1}, a_{2}\right)$ yields $\sigma((1-\lambda) x+\lambda y)>\sigma(x)$.

Another interesting property of $\sigma(v)$ is the following, which states that its logarithm (a separable function) is a concave function.

\section{Proposition 5}

$\ln \sigma(v)$ is concave in $U:=\left\{v \in \mathbb{R}^{n}: v_{j}>-\Delta_{j}(j=\right.$ $1, \ldots, n)\}$.

\section{Proof}

$\ln \sigma(v)=\ln \left[c\left(a_{1}, a_{2}\right) \Pi_{j=1}^{n} F D_{j}\left(v_{j}\right)\right]=\ln c\left(a_{1}, a_{2}\right)+$ $\sum_{j=1}^{n} \ln F D_{j}\left(v_{j}\right)$. Since $c\left(a_{1}, a_{2}\right)$ is kept constant and the $F D_{j}($.$) are concave (Proposition 3), we$ obtain a sum of concave functions.

\section{A.2. Type 3 constraint}

We now consider the case with constraints $\left(k_{1}, \ldots, k_{n}\right)=k \in K, v_{j}=p_{j}+\alpha_{j} / k_{j}$ and $\alpha_{j}^{u} \geq \alpha_{j} \geq \alpha_{j}^{l}$ $(j=1, \ldots, n)$ (Type 3). Next we show that for fixed $q_{j}, p_{j}, \Delta_{j}$ and $\alpha_{j}(j=1, \ldots, n), \sigma(k)$ is a strictly quasiconcave function in the subdomain of $K$ where veto does not occur (Proposition 9). Note also that if any veto occurs or $c\left(a_{1}, a_{2}, k\right)=$ 0 , then we know that the credibility index equals zero. If $c\left(a_{1}, a_{2}, k\right)=1$, then we know that the credibility index equals one. Again, we can restrict the ourselves to the case where the value of the credibility index is unknown (depends on $k \in K$ ), i.e. $\left.\quad c\left(a_{1}, a_{2}, k\right) \in\right] 0,1\left[\right.$ and $v_{j}\left(k_{j}\right)>-\Delta_{j} \quad(j=$ $1, \ldots, n)$ or, equivalentely, $\sigma(k) \in] 0,1[$.

We will prove Proposition 9 by contradiction, showing that $\sigma((1-\lambda) x+\lambda y) \geq \sigma(x) \quad(x, y \in K)$ for a positive small enough $\lambda$, when $0<\sigma(x)<$ $\sigma(y)<1$ (Proposition 8). Before that we prove an auxiliary result (Proposition 7 ) that will provide us a lower bound for $\sigma($.$) .$

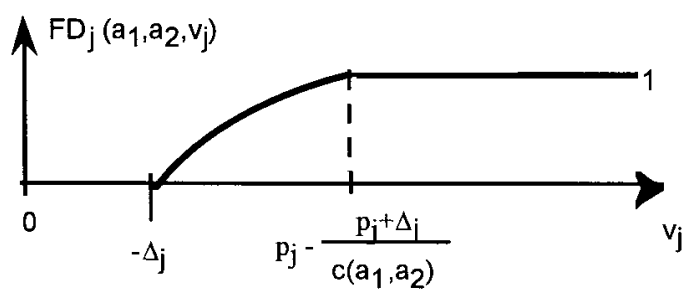

Figure 6. Shape of $F D_{j}\left(v_{j}\right)$. 
Since $\left.c\left(a_{1}, a_{2}, k\right) \in\right] 0,1[$ we can rewrite:

$$
\begin{aligned}
\sigma(k)= & \sigma\left(a_{1}, a_{2}, k\right) \\
= & c\left(a_{1}, a_{2}, k\right) \\
& \times \underset{\substack{j \in\{1, \ldots, n\}: \\
d_{j}\left(a_{1}, a_{2}, k_{j}\right)>c\left(a_{1}, a_{2}, k\right)}}{ } \frac{1-d_{j}\left(a_{1}, a_{2}, k_{j}\right)}{1-c\left(a_{1}, a_{2}, k\right)} \\
= & \frac{c(k)}{(1-c(k))^{n}} \prod_{j=1}^{n} m_{j}(k),
\end{aligned}
$$

where $m_{j}(k)=\min \left\{1-c(k), 1-d_{j}\left(k_{j}\right)\right\}$ (we omit the reference to $\left.\left(a_{1}, a_{2}\right)\right)$.

\section{Proposition 6}

Assuming that no veto occurs, $m_{j}(k)$ is concave in $K$, i.e.

$\left.m_{j}(k)>0 \Rightarrow \forall \lambda \in\right] 0,1[, x, y \in K$,

$m_{j}((1-\lambda) x+\lambda y) \geq(1-\lambda) m_{j}(x)+\lambda m_{j}(y)$.

\section{Proof}

From (3.3) and (4.7c) we have

$d_{j}\left(k_{j}\right)= \begin{cases}0, & \text { if } \Delta_{j} \geq-p_{j} \\ \frac{k_{j}}{\alpha_{j}}\left(-\Delta_{j}-p_{j}\right), & \text { if } \Delta_{j}<-p_{j}\end{cases}$

which is linear on $k_{j}$ whether $\Delta_{j} \geq-p_{j}$ or not. Since $c(k)$ is also linear (Proposition 2), both $1-c(k)$ and $1-d_{j}\left(k_{j}\right)$ are linear. Hence, $m_{j}(k)$, which is the minimum of two linear functions, must be concave.

\section{Proposition 7}

Let $x, y \in K$, such that $m_{j}(x)>0$ and $m_{j}(y)>0$ $(j=1, \ldots, n)$. Then, in a positive neighbourhood of zero small enough,

$\exists \varepsilon \in] 0,1\left[: \forall \lambda \in\left[0, \varepsilon\left[, \prod_{j=1}^{n} m_{j}\left((1-\lambda) x+\lambda_{y}\right)\right.\right.\right.$

$\geq\left(\prod_{j=1}^{n} m_{j}(x)\right)[1+\lambda(r-1)]^{n}$,

with $r=\left(\frac{\prod_{j=1}^{n} m_{j}(y)}{\prod_{j=1}^{n} m_{j}(x)}\right)^{1 / n}$.
Proof

Let $\quad r_{j}=m_{j}(y) / m_{j}(x) \quad(j=1, \ldots, n)$. Since $r_{1}, \ldots, r_{n}>0$ we may define a function

$q(\lambda)=\ln \left(\prod_{j=1}^{n}\left(1-\lambda+\lambda r_{j}\right) / \prod_{j=1}^{n}(1-\lambda+\lambda r)\right)$

( $r$ is defined above). Derivating $q(\lambda)$ yields

$$
\begin{aligned}
\frac{\mathrm{d} q}{\mathrm{~d} \lambda}(\lambda) & =\frac{\mathrm{d} q}{\mathrm{~d} \lambda} \ln \frac{\prod_{j=1}^{n}\left[1+\lambda\left(r_{j}-1\right)\right]}{\prod_{j=1}^{n}[1+\lambda(r-1)]} \\
& =\frac{\mathrm{d} q}{\mathrm{~d} \lambda} \sum_{j=1}^{n} \ln \frac{\left[1+\lambda\left(r_{j}-1\right)\right]}{[1+\lambda(r-1)]} \\
& =\sum_{j=1}^{n} \frac{\mathrm{d}}{\mathrm{d} \lambda} \ln \frac{\left[1+\lambda\left(r_{j}-1\right)\right]}{[1+\lambda(r-1)]} \\
& =\sum_{j=1}^{n} \frac{r_{j}-r}{[1+\lambda(r-1)] \cdot\left[1+\lambda\left(r_{j}-1\right)\right]} .
\end{aligned}
$$

Hence,

$\frac{\mathrm{d} q}{\mathrm{~d} \lambda}(0)=\sum_{j=1}^{n}\left(r_{j}-r\right)=\sum_{j=1}^{n} r_{j}-\sum_{j=1}^{n} r$.

By the well-known Arithmetic-Geometric Mean inequality, given $r_{1}, \ldots, r_{n}>0$,

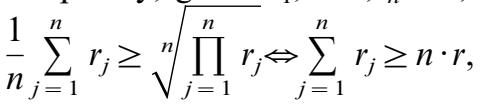

and the equality holds iff $r_{1}=r_{2}=\cdots=r_{n}=r$. Thus, if neither $r_{1}=r_{2}=\cdots=r_{n}=r$ nor $\lambda=0$, then

$\lim _{h \rightarrow 0^{+}} \frac{q(h)-q(0)}{h}=\frac{\mathrm{d} q}{\mathrm{~d} \lambda}(0)>0$.

In these conditions we will have a positive neighbourhood of zero small enough, where $q(\lambda)>$ $q(0)=0$, therefore

$\prod_{j=1}^{n}\left(1-\lambda+\lambda r_{j}\right)>\prod_{j=1}^{n}(1-\lambda+\lambda r)$.

This inequality, together with the concavity of $m_{j}(k)$ (Proposition 6), yields:

$\prod_{j=1}^{n} m_{j}((1-\lambda) x+\lambda y)$

$\geq \prod_{j=1}^{n}\left((1-\lambda) m_{j}(x)+\lambda m_{j}(y)\right)$

$=\left(\prod_{j=1}^{n} m_{j}(x)\right) \cdot \prod_{j=1}^{n}\left(1-\lambda+\lambda r_{j}\right)$

$>\left(\prod_{j=1}^{n} m_{j}(x)\right) \cdot \prod_{j=1}^{n}(1-\lambda+\lambda r)$

$=\left(\prod_{j=1}^{n} m_{j}(x)\right) \cdot \prod_{j=1}^{n}(1-\lambda+\lambda r)^{n}$. 


\section{Proposition 8}

Let $x, y \in K$, such that $0<\sigma(x)<\sigma(y)<1$. Then, in a neighbourhood of zero small enough, $\exists \beta \in] 0,1[: \forall \lambda \in[0, \beta[, \sigma((1-\lambda) x+\lambda y) \geq \sigma(x)$, and if the equality holds then $\lambda=0$.

Proof

Let us define (for a more compact notation) $k(\lambda):=(1-\lambda) x+\lambda y$. Next, we define a function $L(\lambda)$ that (by Proposition 7) does not exceed $\sigma(k(\lambda))$ in a neighbourhood of $\lambda=0$ small enough:

$L(\lambda)=\frac{c(k(\lambda))}{[1-c(k(\lambda))]^{n}}\left(\prod_{j=1}^{n} m_{j}(x)\right)[1+\lambda(r-1)]^{n}$,

with $r=\left(\frac{\prod_{j=1}^{n} m_{j}(y)}{\prod_{j=1}^{n} m_{j}(x)}\right)^{1 / n}$.

Derivating $L(\lambda)$ yields:

$$
\begin{aligned}
\frac{\mathrm{d} L}{\mathrm{~d} \lambda}(\lambda)= & \left(\prod_{j=1}^{n} m_{j}(x)\right) \\
& \times \frac{\mathrm{d}}{\mathrm{d} \lambda}\left\{\frac{c(k(\lambda))}{[1-c(k(\lambda))]^{n}}[1+\lambda(r-1)]^{n}\right\} \\
= & \left(\prod_{j=1}^{n} m_{j}(x)\right) \\
& \cdot \frac{c(k(\lambda))}{[1-c(k(\lambda))]^{n}} \cdot[1+\lambda(r-1)]^{n-1} \\
& \cdot\left\{\left(\frac{\mathrm{d}}{\mathrm{d} \lambda} \ln \frac{c(k(\lambda))}{[1-c(k(\lambda))]^{n}}\right)\right. \\
& \cdot[1+\lambda(r-1)]+n \cdot(r-1)\} .
\end{aligned}
$$

Since $0<\sigma(k)<1$ implies $1>c(k)>0 \quad$ (and $\left.m_{j}(k)>0\right)$, we can define the quotient $Q=$ $c(y) / c(x)>0$, where $x, y \in K$. By Proposition 2, $c((1-\lambda) x+\lambda y)=(1-\lambda) \cdot c(x)+\lambda \cdot c(y)$. Hence, $c(k(\lambda))=c(x)(1-\lambda+\lambda Q)$. Let us also define

$$
\begin{aligned}
p(\lambda)= & \left(\prod_{j=1}^{n} m_{j}(x)\right) \\
& \cdot \frac{c(k(\lambda))}{[1-c(k(\lambda))]^{n}} \cdot[1+\lambda(r-1)]^{n-1}
\end{aligned}
$$

and rewrite

$$
\frac{\mathrm{d} L}{\mathrm{~d} \lambda}(\lambda)=p(\lambda) \cdot\left\{\left(\frac{\mathrm{d}}{\mathrm{d} \lambda} \ln [c(x)(1-\lambda+\lambda Q)]\right.\right.
$$

$$
\begin{aligned}
& \left.-n \frac{\mathrm{d}}{\mathrm{d} \lambda} \ln [1-c(x)(1-\lambda+\lambda Q)]\right) \\
& {[1+\lambda(r-1)]+n \cdot(r-1)\} } \\
= & p(\lambda) \cdot\left\{\left(\frac{Q-1}{1-\lambda+\lambda Q}\right.\right. \\
& \left.-n \frac{-c(x)(Q-1)}{1-c(x)(1-\lambda+\lambda Q)}\right) \cdot[1+\lambda(r-1)] \\
& +n \cdot(r-1)\} .
\end{aligned}
$$

Therefore,

$$
\begin{aligned}
\frac{\mathrm{d} L}{\mathrm{~d} \lambda}(0) & =p(0) \cdot\left\{Q-1+n \frac{c(x)(Q-1)}{1-c(x)}+n \cdot(r-1)\right\} \\
& =\sigma(x) \cdot\left\{Q-1+n \frac{c(x)(Q-1)}{1-c(x)}+n \cdot(r-1)\right\} .
\end{aligned}
$$

Now note that

$$
\begin{aligned}
\sigma(x) & <\sigma(y) \Leftrightarrow \frac{c(x)}{(1-c(x))^{n}} \prod_{j=1}^{n} m_{j}(x) \\
& <\frac{Q \cdot c(x)}{(1-Q \cdot c(x))^{n}} \prod_{j=1}^{n}\left[m_{j}(x) \cdot r_{j}\right] \\
& \Leftrightarrow r>\sqrt[n]{\frac{1}{Q}} \cdot \frac{1-Q \cdot c(x)}{1-c(x)},
\end{aligned}
$$

which yields (since $\sigma(x)>0$ )

$$
\begin{aligned}
\frac{\mathrm{d} L}{\mathrm{~d} \lambda}(0) & >\sigma(x) \cdot\left\{Q-1+n \frac{c(x)(Q-1)}{1-c(x)}\right. \\
& \left.+n \cdot\left(\sqrt[n]{\frac{1}{Q}} \cdot \frac{1-Q \cdot c(x)}{1-c(x)}-1\right)\right\} .
\end{aligned}
$$

Consider now the second term of this product:

$$
\begin{aligned}
& f_{\text {aux }}(Q)=Q-1+n \frac{c(x)(Q-1)}{1-c(x)} \\
& +n \cdot\left(\sqrt[n]{\frac{1}{Q}} \cdot \frac{1-Q \cdot c(x)}{1-c(x)}-1\right) .
\end{aligned}
$$

Since

$$
\begin{aligned}
\frac{\mathrm{d}}{\mathrm{dQ}} f_{\mathrm{aux}}(Q)= & 1-\frac{1}{Q} \cdot \sqrt[n]{\frac{1}{Q}} \cdot \frac{1-Q \cdot c(x)}{1-c(x)} \\
& +n \frac{c(x)}{1-c(x)}\left(1-\sqrt[n]{\frac{1}{Q}}\right)
\end{aligned}
$$

is negative for $Q<1$, positive for $Q>1$ and null for $Q=1$, the minimum of $f_{\text {aux }}(Q)$ is $f_{\text {aux }}(1)=0$. Thus, $f_{\text {aux }}(Q) \geq 0$, which in turn implies that 

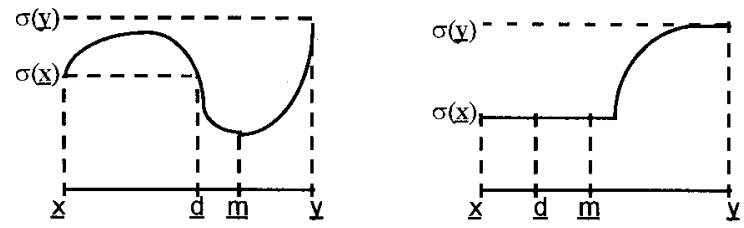

Figure 7. Two continuous not strictly quasiconcave functions: the function on the left is not quasiconcave; the function on the right is quasiconcave but strictly quasiconcave.

$\frac{\mathrm{d} L}{\mathrm{~d} \lambda}(0)=\lim _{h \rightarrow 0^{+}} \frac{L(h)-L(0)}{h}>0$.

In summary, $\exists \delta \in] 0,1[: \forall \lambda \in[0, \delta[, L(\lambda) \geq L(0)$, and the equality only holds for $\lambda=0$. Since $L(0)=\sigma(x)$ and $\exists \varepsilon \in] 0,1[: \forall \lambda \in[0, \varepsilon[, \sigma(k(\lambda)) \geq$ $L(\lambda)$, we conclude that for $\beta=\min \{\delta, \varepsilon\}$, $\forall \lambda \in[0, \beta[, \quad \sigma((1-\lambda) x+\lambda \mathrm{y}) \geq \sigma(x)$, and if the equality holds then $\lambda=0$.

\section{Proposition 9}

Let $I=\{x \in K: \sigma(x) \in] 0,1]\}$. Then $\sigma(x)$ is strictly quasiconcave in $I$, i.e. $\forall \lambda \in] 0,1[, x, y \in I$, with $\sigma(x) \neq \sigma(y), \sigma((1-\lambda) x+\lambda y)>\min \{\sigma(x), \sigma(y)\}$.

\section{Proof}

Without loss of generality, assume that $\sigma(x)<$ $\sigma(y)$. Suppose that the proposition is false and let $\left.\lambda_{m} \in\right] 0,1[$ be a value associated with a point $m=$ $\left(1-\lambda_{m}\right) x+\lambda_{m} y$, such that $\sigma(m) \leq \sigma(x)$. Since $\sigma($.$) is continuous, there must exist a point d$, which is either $x$ or a point between $x$ and $m$, such that $\sigma(d)=\sigma(x)$ and $\sigma($.$) does not increase in the$ neighbourhood of $d$ in the direction of $m$ (see Figure 7). However, Proposition 8, states that $\sigma($.) should increase in the neighbourhood of $d$ in the direction of $y$, since $\sigma(d)<\sigma(y)$. We arrive at a contradiction, hence the proposition must be true.

\section{REFERENCES}

Avis, D. and Fukuda, K., 'A pivoting algorithm for convex hulls and vertex enumeration of arrangements and polyhedra', Discrete Comput. Geomet., 8, 295-313 (1992).

Bazaraa, M.S., Sherali, H.D. and Shetty, C.M., Nonlinear Programming: Theory and Algorithms, 2nd edition, New York: Wiley, 1993.

Dias, L.C. and Clímaco, J.N., 'Shortest path problems with partial information: models and algorithms for detecting dominance', Eur. J. Oper. Res. (1998a) (forthcoming).

Dias, L.C. and Clímaco, J.N. 'ELECTRE TRI and group decision aiding: an analytic study on the robustness of the conclusions', Research report, Management Science Series, No. 3/98, Faculty of Economics, University of Coimbra, 1998b.

Dias, L.C., Costa, J.P. and Clímaco, J.N., 'Conflicting criteria, cooperating processors-some experiments on implementing a multicriteria decision support method on a parallel computer', Comput. Oper. Res., 24, 805-817 (1997).

Fishburn, P.C., Decision and Value Theory, New York: Wiley, 1964.

French, S., 'Uncertainty and imprecision: modelling and analysis', J. Oper. Res. Soc., 46, 70-79 (1995).

Gromicho, J.A.S., Quasiconvex Optimization and Location Theory, Applied Optimization 9, Dordrecht: Kluwer, 1998.

Hazen, G.B., 'Partial information, dominance and potential optimality in multiattribute utility theory', Oper. Res., 34(2), 297-310 (1986).

Henggeler Antunes, C. and Clímaco, J.N., 'Sensitivity analysis in MCDM using the weight space', Oper. Res. Lett., 12, 187-196 (1992).

Horst, R. and Tuy, H., Global Optimization: Deterministic Approaches, 3rd edition, Berlin: Springer, 1996.

Hurson, Ch. and Zopounidis, C., 'On the use of multicriteria decision aid methods to portfolio selection', in Clímaco, J. (ed.), Multicriteria Analysis, Berlin: Springer, 1997, pp. 496-507.

Kampke, T., 'Sensitivity analysis for assessing preferentially independent order relations', Comput. Oper. Res., 23(12), 1119-1130 (1996).

Kirkwood, C.W, and Sarin, R.K., 'Ranking with partial information: a method and an application', Oper. Res., 33(1), 38-48 (1985).

Kouvelis, P. and Yu, G., Robust Discrete Optimization and its Applications, Dordrecht: Kluwer, 1997.

Lemaréchal, C., 'Nondifferentiable optimization', in Nemhauser, G.L. et al. (eds), Handbooks in Operations Research/Management Science, Vol. 1, Amsterdam: Elsevier, 1989, pp. 529-572.

Maystre, L.-Y., Pictet, J. and Simos, J., Méthodes Multicritères ELECTRE, Lausanne: Presses Polytechniques et Universitaires Romandes, 1994.

Mousseau, V., Problémes liés a l'evaluation de l'importance relative des critères en aide multicritère à la décision: refléxions théoriques, experimentation et implémentation informatique, $\mathrm{PhD}$ Thesis, Université Paris-Dauphine, 1993.

Rinnooy Kan, A.H.G. and Timmer, G.T., 'Global optimization', in Nemhauser, G.L. et al. (eds), Handbooks in Operations Research/Management Science, Vol. 1, Amsterdam: Elsevier, 1989, pp. 631-662.

Rios Insua, D. and French, S., 'A framework for sensitivity analysis in discrete multi-objective decision-making', Eur. J. Oper. Res., 54, 176-190 (1991). 
Roy, B., 'The outranking approach and the foundations of ELECTRE methods', in Bana e Costa, C.A. (ed), Readings in Multiple Criteria Decision Aid, Berlin: Springer-Verlag, 1990.

Roy, B., 'Un chainon manquant en RO-AD: les conclusions robustes', Cahier du LAMSADE, No. 144, Université de Paris Dauphine, 1997.

Roy, B. and Bouyssou, D., 'Main sources of inaccurate determination, uncertainty and imprecision in deci- sion models', Math. Comput. Model., 12, 1245-1254 (1989).

Roy, B. and Bouyssou, D., Aide Multicritère a la Décision: Méthodes et Cas, Paris: Economica, 1993.

Vincke, Ph., 'Robust solutions and methods on decision aid' (1997) (forthcoming).

Weber, M., 'Decision making with incomplete information', Eur. J. Oper. Res., 28, 44-57 (1987). 